\title{
Polyomavirus Nephropathy: Ten-Year Experience
}

\author{
J.S. Costa ${ }^{a, *}$, E. Ferreira ${ }^{a}$, R. Leal ${ }^{a}$, N. Bota ${ }^{b}$, C. Romãozinho ${ }^{a}$, V. Sousa ${ }^{c}$, C. Marinho ${ }^{c}$, L. Santos ${ }^{a}$, \\ F. Macário ${ }^{a}$, R. Alves ${ }^{a}$, J. Pratas ${ }^{a}$, M. Campos ${ }^{a}$, and A. Figueiredo \\ ${ }^{a}$ Department of Nephrology, Coimbra Hospital and Universitary Center, Coimbra, Portugal; ${ }^{\circ}$ Department of Nephrology, Clínica Sagrada \\ Esperança, Luanda, Angola; 'Department of Anatomic Pathology, Coimbra Hospital and Universitary Center, Coimbra, Portugal; and \\ ${ }^{\mathrm{d}}$ Department of Urology and Kidney Transplantation, Coimbra Hospital and Universitary Center, Coimbra, Portugal
}

\begin{abstract}
Background. Polyomavirus nephropathy (BKVN) is an important cause of chronic allograft dysfunction (CAD). Recipient determinants (male sex, white race, and older age), deceased donation, high-dose immunosuppression, diabetes, delayed graft function (DGF), cytomegalovirus infection, and acute rejection (AR) are risk factors. Reducing immunosuppression is the best strategy in BKVN. The objective of our study was to evaluate $\mathrm{CAD}$ progression after therapeutic strategies in BKVN and risk factors for graft loss (GL).

Methods. Retrospective analysis of 23 biopsies, from patients with CAD and histological evidence of BKVN, conducted over a period of 10 years. Glomerular filtration rate was $<30 \mathrm{~mL} / \mathrm{min}$ in 16 patients at the time of the BKVN diagnosis.

Results. BKVN was histologically diagnosed in 23 recipients (19 men, 4 women). All patients were white, with age of $51.2 \pm 12.1$ years (6 patients, age $>60$ years), and 22 had a deceased donor. Diabetes affected 4 patients, DGF occurred in 3, cytomegalovirus infection in 2, and AR in 15. All patients were medicated with calcineurin inhibitors (CNI) $(95.7 \%$ tacrolimus) and corticoids, and 16 also received an antimetabolite. One year after antimetabolite reduction/discontinuation and/or CNI reduction/switching and/or antiviral agents, graft function was decreased in 11 patients, increased/stabilized in 10, and unknown in 2. GL occurred in 9 patients. Older age (hazard ratio, 1.76; 95\% confidence interval, 0.94-3.28) and DGF (hazard ratio, 2.60; $95 \%$ confidence interval, 0.54-12.64) were the main risk factors for GL. The lower GFR at the time of the BKVN diagnosis was associated with an increased risk of initiation of dialysis.
\end{abstract}

Conclusions. GL occurred in $39.1 \%$ of patients with BKVN and DGF; older age and lower GFR at the time of diagnosis were important risk factors. Early diagnosis of BKVN is essential to prevent GL.

$\mathbf{B}^{\mathrm{k}}$ K VIRUS (BKV) is a non-enveloped DNA virus of the polyomaviridae family, and $80 \%$ to $90 \%$ of the general population is seropositive for BKV [1]. Primary infection usually occurs in the first decade of life, probably by respiratory or oral transmission, and then becomes latent mainly in the urinary tract [2]. Under sustained and intensive immunosuppression, the reactivation of latent BKV can occur, leading to polyomavirus-associated nephropathy (BKVN) in $1 \%$ to $10 \%$ of kidney transplant patients [2], most frequently in the first 2 years after transplantation [3]. This progressively affects graft function and increases the risk of chronic allograft dysfunction (CAD) and graft loss (GL) [2], more often with BKVN late diagnosis. BKV incidence has increased since the mid-90s with the use of new powerful immunosuppressor treatments [4]. Because effective and safe antiviral therapies are lacking [2,3],

*Address correspondence to Joana Silva Costa, Centro Hospitalar e Universitário de Coimbra-Hospitais da Universidade de Coimbra, Serviço de Nefrologia, Praceta Prof. Mota Pinto, Coimbra 3000-075, Portugal. E-mail: joana.c.s.costa@gmail.com

$0041-1345 / 17$

http://dx.doi.org/10.1016/j.transproceed.2017.01.072 


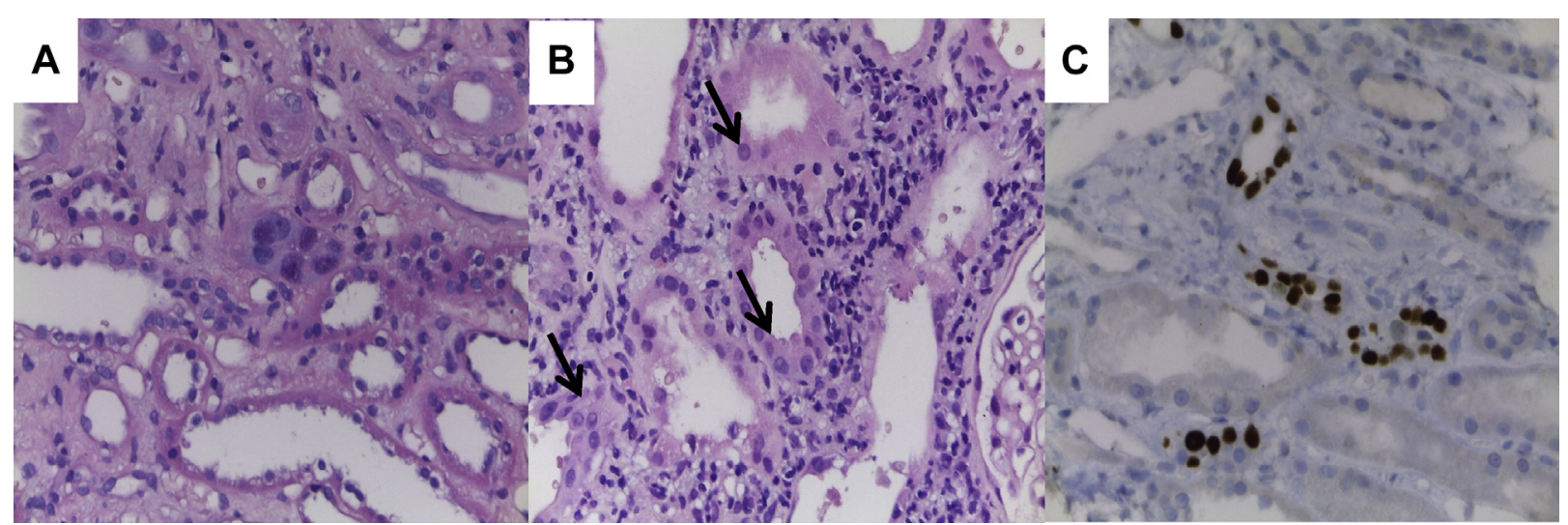

Fig 1. BK virus nephropathy in kidney allograft biopsies. (A) Tubular epithelial cells with large, irregular nuclei, nucleoli, basophilic chromatin, and vesicular changes; hematoxylin and eosin (HE) stain, magnification $\times 400$. (B) Polyomavirus intranuclear inclusions (arrows) in the tubular epithelial cells; HE stain, magnification $\times 400$. (C) Characteristic nuclear staining reaction (antibody directed against the BKV SV40 T antigen) demonstrated by immunohistochemistry; magnification $\times 400$.

identification of risk factors and screening for BKV replication is essential.

Some of the risk factors for BKVN are donor determinants (HLA mismatches and deceased donation), recipient determinants (older age, male sex, white race), and modulating factors after transplantation (acute rejection, delayed graft function [DGF], steroid exposure, lymphocyte-depleting antibody medication, high dose of immunosuppressive medication, and tacrolimus-mycophenolic acid compared with cyclosporinemycophenolic acid or mTOR inhibitor combinations) [2].

Viremia by polymerase chain reaction (PCR) detection is the recommended screening test for BKVN. A level superior to $10^{4}$ copies/mL is associated with a $93 \%$ specificity [2] and can establish "presumptive BKVN" [2,5]. However, only histological findings of BKVN (cytopathic tubular epithelial cell changes, intra-nuclear viral inclusion bodies in tubular epithelial cells, and immunohistochemistry detection of monoclonal antibodies directed against BKV SV40 T antigen) in the allograft biopsy allows its definitive diagnosis $[2,5]$.
Viremia can guide a stepwise reduction of immunosuppression, which appears to be the best therapeutic strategy to delay the progression of CAD. Most studies consider that a period of more than 12 weeks of reduced immunosuppression is required to significantly reduce viral load levels [5]. The effectiveness of additional strategies such as switching immunosuppressive medication (eg, calcineurin inhibitors (CNI) to mTOR inhibitor), with no randomized, controlled trial recommending one over the other, or use of antiviral therapy (quinolone or leflunamide), are still not proven [2,3].

The objective of the study was to evaluate $\mathrm{CAD}$ progression after therapeutic strategies in BKVN and risk factors for GL.

\section{METHODS}

We retrospectively reviewed 23 kidney allograft biopsies from patients with CAD, between January 2005 and December 2015. All patients had histological evidence of BKVN, with intra-nuclear viral inclusions in the tubular epithelial cells, stained with hematoxylin-
A

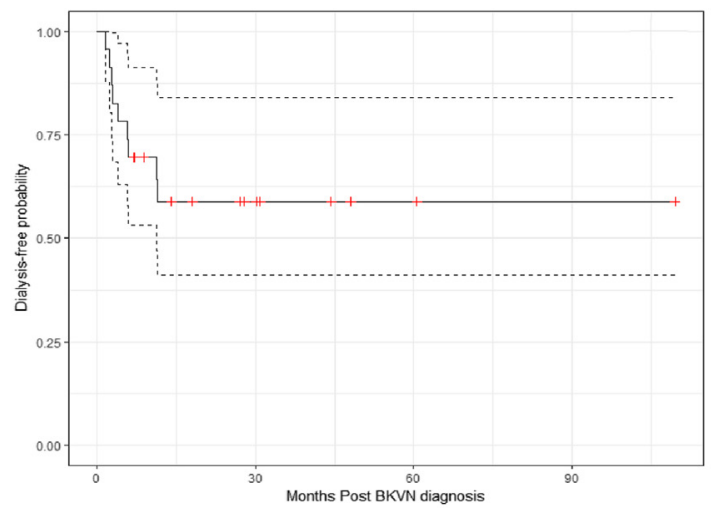

B

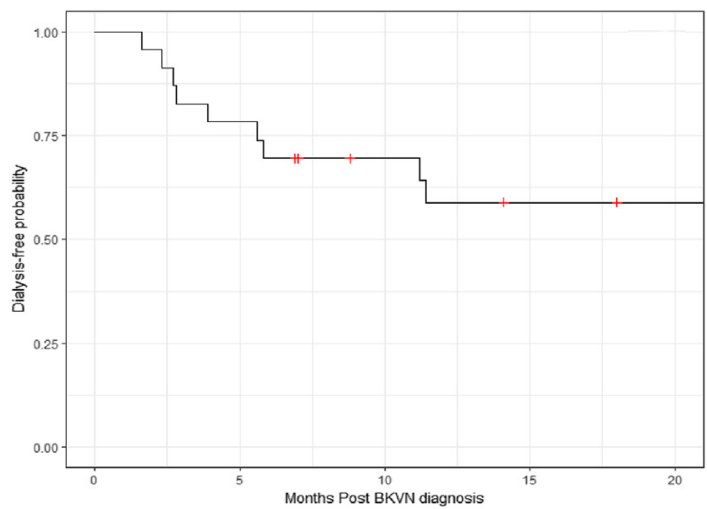

Fig 2. Kaplan-Meier estimate of time to dialysis after BKVN diagnosis (including $95 \% \mathrm{Cls}$ ), from the time of BKVN diagnosis until the end of the study (A) and for the first 20 months after the diagnosis of BKVN (B). 
Table 1. Individual Characteristics (1)

\begin{tabular}{|c|c|c|c|c|c|c|}
\hline Id & G & Age (Years) & Donor & DGF & $\begin{array}{l}\text { Immunosuppression } \\
\text { at Diagnosis }\end{array}$ & Therapeutic Strategy \\
\hline 1 & M & 56 & $\mathrm{D}$ & No & $\mathrm{FK}+\mathrm{PDN}$ & Quinolone + antimetabolite reduction/discontinuation + conversion FK to EVE \\
\hline 2 & M & 55 & $\mathrm{D}$ & No & $\mathrm{MY}+\mathrm{FK}+\mathrm{PDN}$ & Quinolone + antimetabolite reduction/discontinuation + conversion FK to EVE \\
\hline 3 & M & 63 & $\mathrm{D}$ & No & $\mathrm{MY}+\mathrm{FK}+\mathrm{PDN}$ & Quinolone + CNI reduction + antimetabolite reduction/discontinuation \\
\hline 4 & $\mathrm{~F}$ & 42 & $\mathrm{D}$ & No & $\mathrm{FK}+\mathrm{PDN}$ & Quinolone + antimetabolite reduction/discontinuation + conversion FK to EVE \\
\hline 5 & M & 53 & $\mathrm{D}$ & No & $\mathrm{MY}+\mathrm{FK}+\mathrm{PDN}$ & antimetabolite reduction/discontinuation + conversion FK to EVE \\
\hline 6 & M & 31 & $\mathrm{D}$ & No & $\mathrm{MY}+\mathrm{FK}+\mathrm{PDN}$ & $\mathrm{CNI}$ reduction \\
\hline 7 & M & 25 & $\mathrm{D}$ & Yes & $\mathrm{MMF}+\mathrm{FK}+\mathrm{PDN}$ & $\mathrm{CNI}$ reduction + antimetabolite reduction/discontinuation \\
\hline 8 & $\mathrm{~F}$ & 38 & D & No & $\mathrm{MMF}+\mathrm{FK}+\mathrm{PDN}$ & antimetabolite reduction/discontinuation $+\mathrm{CNI}$ reduction \\
\hline 9 & M & 65 & $\mathrm{D}$ & No & $\mathrm{FK}+\mathrm{PDN}$ & Quinolone $+\mathrm{CNI}$ reduction + antimetabolite reduction/discontinuation \\
\hline 10 & M & 34 & $\mathrm{D}$ & No & $\mathrm{FK}+\mathrm{PDN}$ & conversion FK to EVE \\
\hline 11 & M & 53 & $\mathrm{D}$ & No & $\mathrm{FK}+\mathrm{PDN}$ & Quinolone $+\mathrm{CNI}$ reduction + antimetabolite reduction/discontinuation \\
\hline 12 & $\mathrm{~F}$ & 48 & $\mathrm{D}$ & No & $\mathrm{MY}+\mathrm{FK}+\mathrm{PDN}$ & $\begin{array}{l}\text { Quinolone }+ \text { Leflunamide }+ \text { antimetabolite reduction/discontinuation }+ \text { conversion } \\
\text { FK to EVE }\end{array}$ \\
\hline 13 & M & 52 & D & No & $\mathrm{MMF}+\mathrm{FK}+\mathrm{PDN}$ & Quinolone + antimetabolite reduction/discontinuation + conversion FK to CyP \\
\hline 14 & $\mathrm{~F}$ & 61 & $\mathrm{D}$ & No & $\mathrm{MY}+\mathrm{FK}+\mathrm{PDN}$ & Quinolone + antimetabolite reduction/discontinuation + conversion FK to CyP \\
\hline 15 & M & 54 & $\mathrm{~L}$ & No & $\mathrm{MY}+\mathrm{FK}+\mathrm{PDN}$ & $\begin{array}{l}\text { Quinolone + Leflunamide + CNI reduction + antimetabolite reduction/ } \\
\text { discontinuation }\end{array}$ \\
\hline 16 & M & 47 & $\mathrm{D}$ & No & $\mathrm{MMF}+\mathrm{CyP}+\mathrm{PDN}$ & Leflunamide + antimetabolite reduction/discontinuation + conversion CyP to EVE \\
\hline 17 & M & 46 & $\mathrm{D}$ & Yes & $\mathrm{MMF}+\mathrm{FK}+\mathrm{PDN}$ & Leflunamide + antimetabolite reduction/discontinuation + conversion FK to CyP \\
\hline 18 & M & 48 & $\mathrm{D}$ & No & $\mathrm{MY}+\mathrm{FK}+\mathrm{PDN}$ & Leflunamide + CNI reduction + antimetabolite reduction/discontinuation \\
\hline 19 & M & 44 & $\mathrm{D}$ & No & $\mathrm{MMF}+\mathrm{FK}+\mathrm{PDN}$ & Quinolone + conversion FK to CyP + antimetabolite reduction/discontinuation \\
\hline 20 & M & 66 & $\mathrm{D}$ & No & $\mathrm{MMF}+\mathrm{FK}+\mathrm{PDN}$ & antimetabolite reduction/discontinuation \\
\hline 21 & M & 74 & $\mathrm{D}$ & Yes & $\mathrm{FK}+\mathrm{PDN}$ & Quinolone $+\mathrm{CNI}$ reduction + antimetabolite reduction/discontinuation \\
\hline 22 & M & 56 & $\mathrm{D}$ & No & $\mathrm{AZA}+\mathrm{FK}+\mathrm{PDN}$ & $\mathrm{CNI}$ reduction \\
\hline 23 & M & 66 & D & No & $\mathrm{FK}+\mathrm{PDN}$ & $\mathrm{CNI}$ reduction + antimetabolite reduction/discontinuation \\
\hline
\end{tabular}

Abbreviations: AZA, azathioprine; CNI, calcineurin inhibitor; CyP, cyclosporine; D, deceased donor; DGF, delayed graft function; EVE, everolimus; F, female; FK, tacrolimus; G, gender; L, living donor; M, male; MFA, mycophenolic acid; MMF, mycophenolate mofetil; PDN, prednisone.

eosin, and positive polyomavirus SV40 large T-antigen expression, by immunohistochemistry (Fig 1). Data regarding transplant recipient and donor demographics, plasma BKV (viremia) values, glomerular filtration rate (GFR) and immunosuppressive medication at the time of the biopsy, BKVN therapeutic strategy and posterior viremia, and graft function evolution were collected. At the time of the biopsy, viremia was $>10^{4}$ copies $/ \mathrm{mL}$ in 13 patients, $\leq 10^{4}$ copies $/ \mathrm{mL}$ in 5 patients, and not tested in 5 patients; plasmatic creatinine was $3.3 \pm 1.2 \mathrm{mg} / \mathrm{dL}$ and GFR (MDRD) was $<30 \mathrm{~mL} / \mathrm{min}$ in 16 patients. BKV DNA was detected through the use of a quantitative PCR assay and BKV viremia was defined positive when there were $>10^{4}$ copies $/ \mathrm{mL}$ and negative when there were $\leq 10^{4}$ copies $/ \mathrm{mL}$. Date of diagnosis of BKVN was defined as the date of the first biopsy with positive immunohistochemistry for BKV.

The statistical analysis was event-driven, being the event of interest initiation of dialysis. Overall probabilities of not initiating dialysis were obtained and plotted by use of the Kaplan-Meier method and compared by means of the log-rank test when applicable. Univariate analysis was performed to identify potential risk factors, using the Cox proportional hazards model to obtain hazard ratios (HR). The Fisher exact test was also calculated, when possible, to assess the individual predictive value of each factor regardless of the time factor. All statistical analysis were conducted using R Statistical Software version 3.2.5.

\section{RESULTS}

BKVN was histologically diagnosed in 23 kidney recipients during the 10 -year period analyzed, $17.8 \pm 33.3$ months after transplant. Figure 2 shows that the risk of
GL is higher during the first 12 months after the diagnosis of BKVN. The individual characteristics of these patients are shown in Tables 1 and 2. All patients were white, mean age was $51.2 \pm 12.1$ years ( 6 patients were $>60$ years of age), and the majority (19 patients) were men. Almost all patients had a deceased donor (22 patients), and basiliximab was the most commonly used induction therapy agent (18 patients). At the time of the biopsy, all patients were medicated with CNI (22 patients with tacrolimus [FK], 1 patient with cyclosporine [CyP]) and corticoids, in combination with an antimetabolite in 16 patients (mycophenolic acid in 8 patients, mycophenolate mofetil in 7 patients, and azathioprine in 1 patient). Diabetes affected 4 patients: 1 patient had type 2 diabetes mellitus previous to the transplant and 3 patients were diagnosed with new-onset diabetes after transplantation. DGF occurred in 3 patients. There was cytomegalovirus infection in 1 patient at the time of the biopsy and in 1 patient before the biopsy. Acute rejection (AR) was diagnosed in 3 patients at the time of BKVN diagnosis (one with borderline rejection and one with T-cellmediated rejection type IB, both medicated with methylprednisolone pulse therapy [MTP]; one with acute antibody-mediated rejection, medicated with immunoglobulin therapy, plasmapheresis, and rituximab) and in 12 patients before the biopsy ( 6 patients medicated with MTP, 3 patients medicated with MTP + thymoglobulin, and 3 non-medicated patients). 
Table 2. Individual Characteristics (2)

\begin{tabular}{|c|c|c|c|c|c|c|c|c|}
\hline Id & $\begin{array}{l}\text { Diabetes } \\
\text { Mellitus }\end{array}$ & $\begin{array}{c}\text { CMV } \\
\text { Infection }\end{array}$ & $\begin{array}{c}\text { Acute } \\
\text { Rejection }\end{array}$ & $\begin{array}{c}\text { GFR Group at } \\
\text { Diagnosis }(\mathrm{mL} / \mathrm{min})\end{array}$ & $\begin{array}{c}\text { Viremia }>10^{4} \text { Copies } / \mathrm{mL} \\
\text { at Diagnosis }\end{array}$ & Viremia After Therapy & $\begin{array}{c}\text { Decreased Graft } \\
\text { Function (1 Year } \\
\text { After Therapy) }\end{array}$ & $\mathrm{GL}$ \\
\hline 1 & Yes & No & No & $15-29$ & Yes & Decreased & Increased & No \\
\hline 2 & No & No & No & $15-29$ & Yes & Decreased & U & No \\
\hline 3 & Yes & No & Yes & $15-29$ & Yes & U & $U$ & No \\
\hline 4 & No & No & Yes & $15-29$ & Yes & Negative & Increased & No \\
\hline 5 & No & $\mathrm{D}$ & Yes & $30-44$ & No & Decreased & Stabilized & No \\
\hline 6 & No & No & No & $45-59$ & U & Negative & Yes & No \\
\hline 7 & No & No & Yes & $30-44$ & $U$ & U & Increased & No \\
\hline 8 & No & No & Yes & $30-44$ & No & Negative & Stabilized & No \\
\hline 9 & No & No & Yes & $<15$ & No & Decreased & Yes & Yes \\
\hline 10 & No & No & Yes & $30-44$ & Yes & Decreased & Stabilized & No \\
\hline 11 & No & No & No & $15-29$ & Yes & Negative & Increased & No \\
\hline 12 & No & No & No & $15-29$ & Yes & Increased & Stabilized & No \\
\hline 13 & No & No & Yes & $15-29$ & No & Negative & Increased & No \\
\hline 14 & No & PrD & No & $<15$ & Yes & Decreased & Yes & Yes \\
\hline 15 & No & No & Yes & $30-44$ & Yes & Decreased & Yes & No \\
\hline 16 & No & No & No & $30-44$ & Yes & Decreased & Yes & Yes \\
\hline 17 & No & No & No & $15-29$ & $U$ & U & Yes & Yes \\
\hline 18 & No & No & Yes & $<15$ & Yes & $U$ & Yes & Yes \\
\hline 19 & No & No & Yes & $15-29$ & No & U & Yes & Yes \\
\hline 20 & Yes & No & Yes & $<15$ & $\mathrm{U}$ & Decreased & Increased & No \\
\hline 21 & No & No & Yes & $<15$ & Yes & Decreased & Yes & Yes \\
\hline 22 & No & No & Yes & $15-29$ & Yes & U & Yes & Yes \\
\hline 23 & Yes & No & Yes & $15-29$ & $U$ & $U$ & Yes & Yes \\
\hline
\end{tabular}

Abbreviations: CMV, cytomegalovirus; D, at diagnosis of BKVN; DGF, delayed graft function; GFR, glomerular filtration rate; GL, graft loss; PrD, previous to diagnosis of BKVN; U, unknown.

Although not statistically significant, older age (HR, 1.76; $95 \%$ confidence interval [CI], 0.94-3.28) and DGF (HR, 2.60; $95 \%$ CI, 0.54-12.64) Cox regression estimates were high (Table 3), which may denote them as important risk factors for GL.

The lower GFR at the time of the BKVN diagnosis was associated with an increased risk of initiation of dialysis.
Table 3 shows that patients with GFR 15 to $29 \mathrm{~mL} / \mathrm{min}$ were approximately one-third less likely of starting dialysis than patients with GFR $<15 \mathrm{~mL} / \mathrm{min}$. Kaplan-Meier plots (Fig 3) also support these findings.

The immunosuppressive medication was reduced in all patients, and at least one of the following therapeutic

Table 3. Risk Factors for Initiation of Dialysis

\begin{tabular}{|c|c|c|c|c|}
\hline Factor & $\mathrm{n}(\mathrm{N})$ & HR $(95 \% \mathrm{Cl})$ & Cox Log-Likelihood Test & Fisher Exact Test \\
\hline Age (range, 10 years) & NA & $1.76(0.94-3.28)$ & 0.062 & NA \\
\hline Male sex & $8(19)$ & NE & 0.396 & 1 \\
\hline Deceased donor type & $9(22)$ & $\mathrm{NE}$ & 0.294 & .640 \\
\hline \multicolumn{5}{|l|}{ Immunosuppression therapy } \\
\hline FK + PDN & $3(7)$ & $1.32(0.33-5.30)$ & 0.690 & 1 \\
\hline $\mathrm{MY}+\mathrm{FK}+\mathrm{PDN}$ & $2(8)$ & $0.47(0.09-2.29)$ & 0.323 & .399 \\
\hline $\mathrm{MMF}+\mathrm{FK}+\mathrm{PDN}$ & $2(6)$ & $0.678(0.14-3.30)$ & 0.618 & 1 \\
\hline $\mathrm{MMF}+\mathrm{CyP}+\mathrm{PDN}$ & $1(1)$ & $3.14(0.38-26.09)$ & 0.290 & .391 \\
\hline $\mathrm{AZA}+\mathrm{FK}+\mathrm{PDN}$ & $1(1)$ & $10.49(0.95-115.73)$ & 0.055 & .391 \\
\hline Diabetes mellitus & $1(4)$ & $0.64(0.08-5.19)$ & 0.660 & 1 \\
\hline DGF & $2(3)$ & $2.60(0.54-12.64)$ & 0.280 & .538 \\
\hline CMV infection & $1(2)$ & $1.07(0.13-8.57)$ & 0.952 & 1 \\
\hline Acute rejection & $6(15)$ & $1.06(0.26-4.27)$ & 0.932 & 1 \\
\hline Viremia $>10^{4}$ copies $/ \mathrm{mL}$ at the diagnosis & $5(13)$ & $1.12(0.21-5.89)$ & 0.894 & 1 \\
\hline \multicolumn{5}{|l|}{ GFR group at BKVN diagnosis } \\
\hline$<15 \mathrm{~mL} / \mathrm{min}$ & $4(5)$ & $\mathrm{RG}$ & RG & $\mathrm{RG}$ \\
\hline $15-29 \mathrm{~mL} / \mathrm{min}$ & $4(11)$ & $0.35(0.09-1.41)$ & 0.143 & .281 \\
\hline $30-44 \mathrm{~mL} / \mathrm{min}$ & $1(6)$ & $0.12(0.01-1.09)$ & 0.060 & .134 \\
\hline $45-59 \mathrm{~mL} / \mathrm{min}$ & $0(1)$ & NE & 1 & 1 \\
\hline
\end{tabular}

Abbreviations: AZA, azathioprine; CyP, cyclosporine; FK, tacrolimus; MFA, mycophenolic acid; MMF, mycophenolate mofetil; $n$, subjects who had undergone dialysis; N, subjects at risk; NA, not applicable; NE, non-estimable; PDN, prednisone; RG, reference group. 
A

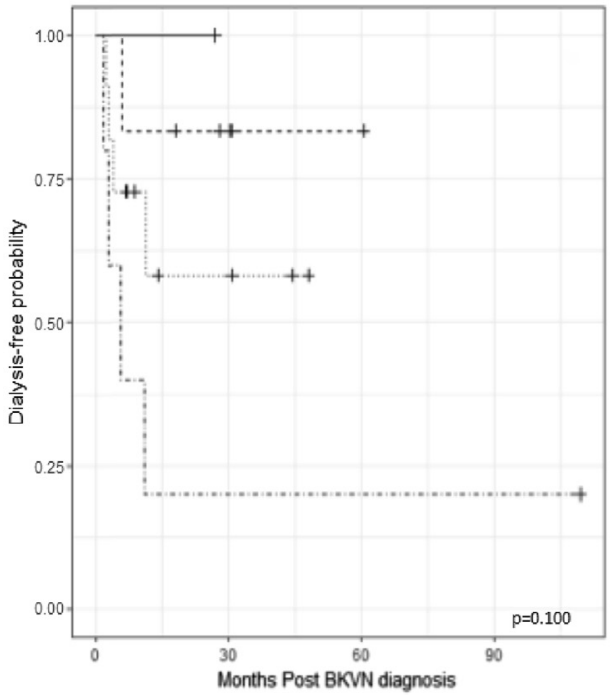

B

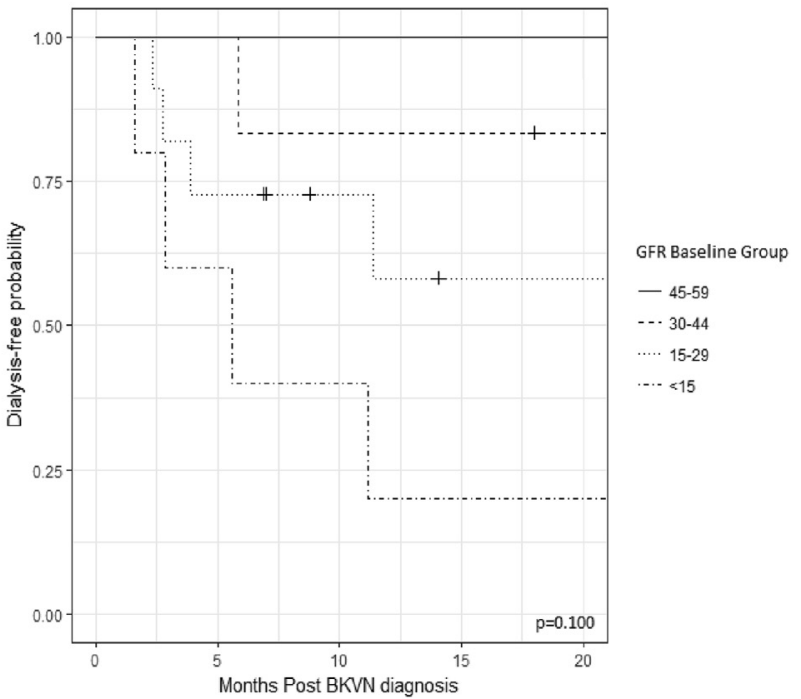

Fig 3. Kaplan-Meier estimate of time to dialysis after BKVN diagnosis by GFR baseline group: from the time of BKVN diagnosis until the end of the study $(\mathbf{A})$ and for the first 20 months after the diagnosis of BKVN (B).

strategies was used: antimetabolite reduction/discontinuation in 20 patients, CNI substitution in 11 patients (FK to everolimus in 6 patients, $\mathrm{FK}$ to $\mathrm{CyP}$ in 4 patients, and $\mathrm{CyP}$ to everolimus in 1 patient), and CNI reduction in 11 patients. Antiviral therapy was used in 15 patients (quinolones during 6 months in 10 patients, oral leflunamide in 3 patients, or both in 2 patients). One year after the initiation of these therapeutic strategies, viremia was increased in 1 patient, reduced in 10 patients, negative $\left(\leq 10^{4}\right.$ copies $\left./ \mathrm{mL}\right)$ in 5 patients, and was not evaluated in 7 patients; allograft function was increased (total recovery in 1 patient, partial recovery in 5 patients) or stabilized in 10 patients $(43.5 \%)$, decreased in 11 patients, and unknown in 2 patients. GL occurred in 9 patients $(39.1 \%), 8$ of them with GFR $<30 \mathrm{~mL} / \mathrm{min}$ (mean GFR of $17.6 \mathrm{~mL} / \mathrm{min}$ ) at the time of biopsy. Of these patients, 4 were $>60$ years of age, 8 were men, 1 was diabetic, 1 had CMV infection, 6 had previous AR (mostly medicated with corticoid + thymoglobulin), and 5 had viremia $>10^{4}$ copies/mL at diagnosis (Tables 1 and 2). The mean postdiagnosis follow-up of the patients was $21.1 \pm 21.6$ months.

\section{DISCUSSION}

In our study, we reviewed renal allograft biopsies that were performed in 23 patients in the setting of CAD and established the diagnosis of BKVN. These patients were white and mainly of male sex, had a deceased donor, and had AR, wellknown risk factors for BKVN. On the other hand, this study revealed that the main risk factors associated with GL were older age and DGF. Although high values of HR have also been seen for MMF + CyP + PDN and AZA + FK + PDN medication at the time of diagnosis of BKVN (Table 3), this finding should be interpreted carefully because only one patient in each group was available to estimate the HR, which may yield inaccurate estimates.

Therapeutic strategies often promote resolution of infection and prevent further decline in the kidney allograft function, but the damage from BKVN may not be reversed, leading to chronic allograft dysfunction [1]. In fact, the results demonstrated that even after strategies had been implemented, a significant percentage of patients had progressive allograft dysfunction. The potential for BKVN to cause high rates of GL is well-documented [6]. We found that $39.1 \%$ of patients (9 patients) had GL after the diagnosis of BKVN, a higher percentage than the $15 \% \mathrm{GL}$ previously described in Wadei et al [6], in which 55 kidney transplant recipients, also with biopsy-proven BKVN, were analyzed during $20 \pm 11$ months of follow-up. Even when patients had increased graft function after the diagnosis of BKVN, more patients had partial recovery than total recovery of the graft function.

This study had some limitations, such as a low number of cases of BKVN histologically diagnosed, because surveillance ("protocol") biopsies were not performed and some patients had contraindications to kidney allograft biopsy. At the time of the biopsy, 16 patients had GFR $<30 \mathrm{~mL} / \mathrm{min}$, and this late diagnosis of BKVN could also be a limitation to the study because these patients were already at a high risk for GL.

Chung et al [7] considered BK viremia to be a useful predictor for BKVN and suggested that its regular monitoring was effective in preventing this pathology. The use of this screening test could promote the early diagnosis of BKVN and early initiation of the therapeutic strategies. The BK viremia screening test was not performed in only 5 patients in this study because this test was not available in the first years of the study. 


\section{CONCLUSIONS}

We found that DGF and older age of the kidney transplant recipients at the time of BKVN diagnosis were important risk factors for GL. Patients with lower GFR at the time of BKVN diagnosis were also at high risk for GL. Therefore, early diagnosis of BKVN is essential to prevent GL.

\section{REFERENCES}

[1] Chon WJ, Aggarwal N, Kocherginsky M, et al. High-level viruria as a screening tool for BK virus nephropathy in renal transplant recipients. Kidney Res Clin Pract 2016;35:176-81.

[2] Hirsch HH, Randhawa P. BK polyomavirus in solid organ transplantation. Am J Transplant 2013;13:179-88.
[3] Kidney Disease Improving Global Outcomes (KDIGO) Transplant Working Group. KDIGO Clinical Practice Guidelines for the Care of Kidney Transplant Recipients. Am J Transplant 2009;9:S44-6.

[4] Lanot A, Bouvier N, Chatelet V, et al. BK virus infections in kidney transplantation. Nephrol Ther 2016;12:76-85.

[5] Nickeleit V, Mihatsch MJ. Polyomavirus nephropathy in native kidneys and renal allografts: an update on as escalating threat. Transplant Int 2006;19:960-73.

[6] Wadei HM, Rule AD, Lewin M, et al. Kidney transplant function and histological clearance of virus following diagnosis of polyomavirus-associated nephropathy. Am J Transplant 2006;6:1025-32.

[7] Chung BH, Hong YA, Kim HG, et al. Clinical usefulness of $\mathrm{BK}$ virus plasma quantitative PCR to prevent $\mathrm{BK}$ virus associated nephropathy. Transplant Int 2012;25:687-95. 